\title{
PESQUisa
}

\section{DESENVOLVIMENTO INFANTIL: UM ESTUDO À PARTIR DO TESTE DENVER II, NA CIDADE DE BARBALHA -CE}

\author{
Hellen Lúcia Cruz Caldas Lins \\ Anamaria Cavalcante e Silva \\ Benedita Cruz Macedo
}

\begin{abstract}
RESUMO
Tem sido grande o avanço na assistência à saúde da criança. Novas tecnologias têm surgido, e vêm sendo utilizadas na recuperação, manutenção e prevenção da saúde infantil. Apesar disso, ainda são complexas as razões de atraso no desenvolvimento infantil observados. Possíveis explicações apontam para a inexistência de sistemas de vigilância, o que dificulta a identificação e acompanhamento das crianças vulneráveis e, a não utilização de instrumentos adequados para triagem de crianças com risco de atraso no desenvolvimento neuropsicomotor. O Objetivo do presente estudo foi avaliar o desenvolvimento de crianças de zero a um ano de vida em 141 crianças acompanhadas no Programa de Saúde da Família no Município de Barbalha-CE. As crianças foram selecionadas aleatoriamente quando da sua viita ao posto de saúde. $\mathrm{O}$ instrumento utilizado para a avaliação do desenvolvimento foi o Teste Denver II, além de um questionário com informações sócio-demográficas.Os resultados nos levaram a crer que o desempenho não se mostrou afetado pelo sexo, pela condição nutricional, renda familiar ou tempo de aleitamento materno exclusivo. $\mathrm{O}$ peso de nascimento, a escolaridade das mães, a assistência pré-natal das mães das crianças e as patologias ocorridas durante a gestação, tipo de parto e estatura atual das crianças afetam o seu desempenho no Teste. Diante dos resultados obtidos ficou evidente a necessidade de se promover e apoiar iniciativas ligadas a programas comunitários de proteção ao desenvolvimento infantil.
\end{abstract}

Palavras-chave: Denver II, Desenvolvimento infantil, Saúde da criança.

Sobre as autoras:

(1) Hellen L. Caldas Lins é Médica, Professora da Universidade Federal do Ceará, Mestre em Saúde da criança e do adolescente pela Universidade Estadual do Ceará (hellenmcruz@hotmail.com).

(2) Anamaria Cavalcante e Silva é Médica, Doutora em Ciências pela Universidade de São Paulo. Diretora da Escola de Saúde Pública do Ceará.

(3) Benedita C. Macedo é Psicóloga, Mestre em Educação e Professora da Universidade Federal do Rio Grande do Norte. 


\section{Introdução}

Nas últimas décadas, tem ocorrido grande avanço na assistência à saúde da criança. Novas tecnologias têm surgido, e vêm sendo utilizadas na recuperação e manutenção da saúde infantil.

No Brasil, país considerado uma das maiores economias do planeta, ainda enfrentamos sérios problemas: $70 \%$ da população encontra-se em situação de pobreza, alguns em miséria absoluta, enquanto $50 \%$ do produto interno bruto estão nas mãos de apenas $10 \%$ da população. A desnutrição infantil, em algumas regiões, atinge índice duas vezes maior que o Ninger. Como se verifica, as desigualdades econômicas aliadas a outras adversidades apresentam-se como um grande desafio (MADEIRA, 1994).

Em setembro de 1978, na cidade de Alma Ata, Capital da República do Kasaquistão, foi realizada a conferência internacional sobre cuidados primários de saúde com o objetivo principal de analisar e propor alternativas que mudassem o quadro de saúde predominante, onde mais da metade da população mundial, não vinha usufruindo dos benefícios de um adequado atendimento.

Os cuidados primários de saúde incluem, segundo a declaração de Alma Ata. A educação no tocante a problemas prevalecentes de saúde e aos métodos, para sua prevenção e controle, promoção na distribuição de alimentos e de nutrição apropriada, provisão adequada de água de boa qualidade e saneamento básico de saúde materno-infantil, inclusive planejamento familiar, imunização contra as principais doenças infecciosas, prevenção e controle de doenças localmente endêmicas, tratamento apropriado de doenças e lesões comuns e fornecimento de medicamentos essenciais (UNICEF, 1978).

Dessa forma, a partir da conferência de Alma Ata, parece estabelecer-se um novo paradigma da saúde, baseando-se essencialmente, em ações de caráter preventivo e de promoção a saúde com a proposta de "saúde para todos no ano 2000" e a estratégia de atenção primária de saúde, que alcançou destaque especial na primeira conferência internacional sobre promoção da saúde (1986), com a promulgação da carta de Ottawa, e que vem se enriquecendo com a série de declarações internacionais periodicamente formulados nas conferências realizadas sobre o tema.

Os principais fatores determinantes do processo saúde-doença, têm sido pouco influenciados pela ação de serviços de saúde, guardando uma maior relação com as condições sócio-econômicas do que com a disponibilidade de serviços. Sendo assim, faz-se necessário que ocorram mudanças fundamentais na organização dos serviços oferecidos, para que os 
sistemas de saúde contemplem o seu papel de promoção, proteção, prevenção, recuperação e reabilitação do indivíduo.(ANDRADE, 1998)

O Ministério da Saúde em 1994, idealizou o Programa Saúde da Família com a finalidade de aprofundar os laços de compromisso e responsabilidade entre as autoridades sanitárias, a instituição, os profissionais de saúde e a população favorecendo assim, a transição do antigo modelo centralizado no hospital para uma assistência com ênfase na promoção à saúde. Devendo dispor de uma equipe capaz de intervir além das práticas curativas habituais, devendo portanto, ser composta por um médico generalista, um enfermeiro, um auxiliar de enfermagem e quatro a seis agentes comunitários de saúde, podendo ser inseridos outros profissionais, de acordo com a necessidade municipal. Cada equipe assumiria a responsabilidade de uma área geográfica onde habitasse de 600 a 1000 famílias, com o máximo de 4500 habitantes. A partir do cadastramento dessas famílias, seria então possível o conhecimento de suas necessidades e o planejamento das ações (BRASIL, 2001).

Com essa proposta surgem em 1994 as primeiras equipes do Programa Saúde da Família. O programa a princípio foi aderido por 55 municípios, colocando em ação 328 equipes e enfrentou vários entraves nesses municípios pioneiros, especialmente advindos da população, um dos grandes equívocos foi acreditar que se tratava de um programa pobre para os pobres, o que não retratava a verdade, pois este encontra-se totalmente voltado para os princípios da universalidade, equidade, integralidade e participação comunitária. Além da resistência da população, houve também forte resistência dos profissionais, pois tratava-se de uma nova concepção de trabalho, onde o profissional "perde" sua posição de superioridade e iguala-se à clientela, além de desenvolver um trabalho prioritariamente multidisciplinar. $\mathrm{O}$ indivíduo já não poderia mais ser visto como uma parte fragmentada, e sim como um todo inserido em um contexto real.

Em 1996 Programa Saúde da Família ganha impulso e aderido por mais de 900 municípios, passando para 847 equipes. No primeiro semestre de 2001 este número era de 12 mil equipes, atuando em mais de 4.500 municípios (BRASIL, 2001).

Das equipes em funcionamento $40 \%$ estão na região Nordeste, sendo que 52\% no Estado do Ceará, 16\% no Estado de Alagoas e 19\% no Estado de Pernambuco (ANDRADE, 1998:19).

Uma pesquisa realizada pelo Ministério da Saúde e FIOCRUZ em todo o Brasil, mostra que até 1999 o Ceará era o estado com maior número de equipes do Programa Saúde da Família. Dos entrevistados, 98\% dos profissionais trabalhando nas equipes da federação, 
acreditam que contribuíram ou estão contribuindo para melhoria das condições de saúde da população (BRASIL, 2001).

O Ministério da Saúde, desde a década de 80, objetivando maximizar o alcance assistencial à saúde infantil, adotou a estratégia de identificar e priorizar ações básicas de comprovada eficácia no controle dos mais relevantes problemas de saúde e que envolvessem reduzida complexidade tecnológica. Esta proposta recebeu a denominação de Ações Básicas na Assistência Integral à Saúde da Criança e se constituiu por cinco ações: Acompanhamento do Crescimento e Desenvolvimento, Aleitamento Materno e Orientação Alimentar para o Desmame, Controle das Doenças Diarréicas, Controle das Infecções Respiratórias Agudas e Controle de Doenças Preveníveis por Imunização (CUNHA, 2000).

O acompanhamento do desenvolvimento infantil torna-se importante por permitir um diagnóstico precoce e, assim, intervir prematuramente, garantindo maior eficácia do tratamento. Baseados em trabalhos clínicos, os autores demonstraram que a eficácia da intervenção precoce é, em alguns casos muito difícil, sobretudo em crianças portadoras de problemas neurológicos como a paralisia cerebral. Mas, as crescentes descobertas sobre o desenvolvimento, quanto mais precocemente se inicia o tratamento, maior é a possibilidade de eficácia do mesmo (NELSON, 1960; MARCONDES, 1994; LEÃO et al., 1998).

Crianças oriundas de famílias de baixa-renda, que vivem em lugares pouco desenvolvidos, como é o caso de muitas cidades do interior do Ceará, tendem a enfrentarem desafios como a sujeição ao nascimento com baixo peso, seja por parto pré-termo, e/ou retardo de crescimento intra-uterino, sofrendo os problemas perinatais decorrentes (VICTORA et al., 1989). Frequentemente vivem em ambientes familiares desfavoráveis, onde a estimulação e o suporte social são inadequados. Esta seqüência de eventos aumenta o risco de atraso em seu desenvolvimento cognitivo, físico e social (BROOKS GUNN, 1990; WERNER, 1997).

Apesar da maioria dos estudos sobre desenvolvimento serem realizados em populações selecionadas de países desenvolvidos (SAIGAL et al.,1991; VOHR, 1991), pesquisas realizadas em países do terceiro mundo têm mostrado importante associação entre o desenvolvimento neuropsicomotor da criança, seu estado nutricional e a situação sócioeconômica da família (GRANTHAM MCGREGOR et al., 1982). Mesmo com as evidências da importância de programas de intervenção precoce (SIMEONSSON et al., 1982) e o sucesso dessas intervenções em crianças de risco em países em desenvolvimento 
(GRANTHAM MCGREGOR et al., 1987), poucos avanços têm ocorrido nesta área. Possíveis explicações para esta lacuna são a complexidade das razões de atraso, a inexistência de sistemas de vigilância, o que dificulta a identificação e acompanhamento das crianças vulneráveis, e a não utilização de instrumentos adequados para triagem de crianças com risco de atraso no desenvolvimento neuropsicomotor (TORBURN, 1990).

$\mathrm{Na}$ prática clínica pediátrica, ainda se observa que crianças expostas aos mesmos fatores ambientais (climáticos, sócio-econômicos, familiares e religiosos), mostram padrões de desenvolvimento bem diferentes. Daí surgem os questionamentos: por que a aquisição de novas habilidades, seja andar, sentar, falar, controlar esfíncteres ocorrem em intervalos de idades tão diferentes? Quais os elementos relacionados ao desenvolvimento são determinantes de variações individuais?

Em 1984, foi publicada pelo Ministério da Saúde uma serie de manuais sobre atenção básica à criança de 0 a 5 anos, dentre os quais o volume que se referia ao acompanhamento do crescimento e desenvolvimento abordava, em sua maior parte, o acompanhamento do crescimento. Posteriormente, alguns desses critérios de acompanhamento foram incluídos no Cartão da Criança.

O desafio do presente estudo foi responder qual o nível de desenvolvimento neuropsicomotor das crianças no seu primeiro ano de vida e quais as variáveis que podem ser preditoras de um melhor desenvolvimento?

O Programa Saúde da Família nos pareceu apropriado para desenvolver esse estudo, por este fornecer as condições mais adequadas de acesso a comunidade de baixa renda para um futuro programa educativo e de educação e saúde que contemple, dente outras atividades, informações sobre o desenvolvimento infantil e seus fatores de risco associados.

\section{Diagnóstico do Desenvolvimento Infantil}

O diagnóstico do desenvolvimento, na literatura especializada, normalmente é analisado sob três aspectos: história médica e história do desenvolvimento, exame físico e neurológico e triagem do desenvolvimento (LEVY e HYMAN, 1993).

A história médica fornece informações sobre a identificação da criança e fatores de riscos biológicos ou ambientais, enquanto que a história do desenvolvimento deverá detectar os marcos desenvolvimentais lentos ou desiguais, em qualquer nível: cognição, fala, linguagem, habilidades motoras, habilidades de adaptação ou psicossociais. 
Os exames físico e neurológico deverão mostrar achados característicos de anormalidades de crescimento, anormalidades congênitas, sinais cutâneos ou organomegalias, que poderão ajudar à determinação da causa dos desvios ou alterações do desenvolvimento.

A triagem do desenvolvimento, através de um instrumento padronizado, poderá identificar precocemente alterações comportamentais. Pesquisadores têm demonstrado que alguns instrumentos são mais confiáveis no esclarecimento diagnóstico que o julgamento clínico (DARGASSIES, 1980; BATSHAW, 1993).

O presente estudo foi realizado com o Teste Denver II. Um instrumento de triagem geral que objetiva fazer a verificação do desenvolvimento de crianças entre zero a seis anos de idade em quatro áreas: pessoal/social, motora/adaptativa, linguagem e motora grosseira.

O Teste de Denver II avalia o desempenho de crianças através de 125 itens ou tarefas, agrupadas em quatro setores que correspondem às quatro grandes áreas do desenvolvimento ou comportamento específicos:1. Conduta pessoal-social; 2. Conduta motora fina-adaptativa; 3. Conduta de linguagem e 4. Conduta motora-grossa.

As instruções para a aplicação e os critérios para avaliação de cada comportamento do Denver-II encontram-se descritas detalhadamente no Manual de Treinamento e Aplicação (ARCHER, et al, 1992).

A importância do Teste de Desenvolvimento de Denver II se justifica tendo em vista que aproximadamente uma em cada oito crianças apresenta alterações do desenvolvimento, que podem interferir de forma significativa em sua qualidade de vida e inclusão na sociedade (GLASCOE et al, 1997). No entanto, o reconhecimento de muitos desses distúrbios é difícil, seja por se apresentarem de forma sutil, seja por raramente estarem associados a alterações morfológicas, ou por se manifestarem incompletamente durante as consultas realizadas. $\mathrm{O}$ prognóstico das crianças com distúrbios do desenvolvimento depende, de forma direta, da identificação e intervenção precoces dessas alterações, o que faz da avaliação do desenvolvimento parte fundamental de toda consulta pediátrica (HALFON et al, 2004).

Nas últimas décadas, o conceito de avaliação do desenvolvimento tem sido substituído pelo de vigilância. De caráter mais amplo, a vigilância engloba, além da prevenção e da detecção de possíveis alterações, a promoção do desenvolvimento da criança, 
que visa, dentre outras ações, a propagação dos conhecimentos atuais relacionados ao desenvolvimento infantil, a antecipação de questões relacionadas a esse processo e a promoção de atitudes que possam favorecê-lo (NICOLL, HUTCHISON, et al. 1990; DWORKIN, 1989; KING, et al. 2004).

O desenvolvimento infantil, portanto, é um processo dinâmico, complexo e de difícil mensuração, em que há grande interdependência entre os vários domínios de funções que o compõem. Nesse sentido, os testes de triagem podem ser utilizados como complementares à avaliação do desenvolvimento (FRANKENBURG, et al., 1992) desde que realizados no contexto da consulta (e não como método isolado) e analisados com base no modo de vida de cada criança.

O Objetivo geral do presente estudo foi avaliar o desenvolvimento neuropsicomotor de crianças com idades entre zero e doze meses completos, atendidas no Programa de Saúde da Família de Barbalha - CE. Para isso, tornou-se importante também: Identificar o desenvolvimento neuropsicomotor segundo o peso ao nascer; Correlacionar o desenvolvimento neuropsicomotor das crianças considerando o estado nutricional, peso e estatura atuais das mesmas; Avaliar o desenvolvimento neuropsicomotor das crianças segundo a renda familiar; Verificar o desenvolvimento neuropsicomotor com relação ao tempo de aleitamento materno exclusivo e xaminar o desenvolvimento neuropsicomotor das crianças segundo a escolaridade das mães.

\section{Metodologia:}

Fizeram parte da amostra 141 crianças de ambos os sexos, de zero a doze meses de idade, atendidas no Programa de Saúde da Família da zona urbana de Barbalha - CE.

Para a coleta dos dados foi utilizado um questionário sócio-econômico, para a entrevista com as mães das crianças e, o Teste de Desenvolvimento Denver II, aplicado às crianças da amostra.

A interpretação final do teste permitiu classificar o desenvolvimento da criança em: Normal: quando não há nenhum tipo de atraso e no máximo um item de risco; Questionável: quando há dois ou mais itens de risco e/ou um ou mais itens de atraso; Não Testável: quando há recusa em um ou mais itens totalmente à esquerda da linha da idade ou recusa em mais de um item cortado pela linha da idade na área de 75 a 90\%. 
Em seguida, houve um retorno aos protocolos de cada criança para processamento dos dados e analise da associação entre as variáveis do estudo, contidas no questionário e o desempenho das crianças no teste Denver II.

\section{Resultados:}

O presente estudo, demonstrou que o desenvolvimento infantil pode ser afetado negativamente pela presença de diversos fatores considerados de risco, durante os primeiros meses de vida da criança. Conhecer e incentivar as pesquisas científicas contribuem para orientar os profissionais quanto a diminuição dessas condições de riscos aos quais estão expostas, principalmente as crianças de baixa renda, como as que fizerem parte da amostra do presente estudo.

Os parágrafos seguintes fazem uma análise sucinta dos resultados, com o propósito de atender aos objetivos do presente estudo.

Com relação ao desenvolvimento neuropsicomotor das crianças entre 0 e 12 meses, atendidas no Programa de Saúde da Família em Barbalha-CE, À partir dos resultados, ficou demonstrado que $63,8 \%$ das crianças apresentaram desempenho normal e 36,2\% apresentaram desempenho questionável e que o sexo não se apresentou como uma variável estatisticamente significante em relação ao desempenho.

Segundo Halpern et al (1996), a elevada proporção de crianças com Teste de Denver II com resultado negativo deve ser interpretada com a devida cautela. Os testes de triagem, apenas alertam para um risco potencial que pode ser confirmado ou não através de um acompanhamento sistemático em outros momentos da vida da criança. Os autores também alertam para o fato de que a avaliação do desenvolvimento em um único momento não permite que se determine, de forma definitiva, um atraso no desenvolvimento da criança. De qualquer forma, os estudos dos autores citados sugerem que, crianças sujeitas a potenciais atrasos de desenvolvimento, podem ser identificadas já ao nascimento, por meio do conhecimento do peso ao nascer. Através desta identificação precoce pode ser possível o estabelecimento de programas de intervenção que visem à prevenção de distúrbios do desenvolvimento.

Quanto ao desenvolvimento neuropsicomotor segundo o peso ao nascer, houve uma diferença estatisticamente significativa em relação ao peso ao nascer quando comparada ao desempenho das crianças da $\operatorname{amostra}\left(\chi^{2}=12,14 ; \mathrm{gl}=3 ; \mathbf{p}=\mathbf{0 , 0 0 3}\right)$. Estes resultados estão de 
acordo com os encontrados por Halpern, Barros, Horta e Victora (1996), que em um estudo sobre o desenvolvimento neuropsicomotor de crianças aos 12 meses de idade no Sul do Brasil. Neste estudo o peso do nascimento esteve significativamente associado com o desempenho neuropsicomotor das crianças. O presente estudo demonstrou a magnitude do efeito do peso ao nascer e das condições da gestação, principalmente quanto ao número de pré-natais das mães sobre o desenvolvimento infantil no primeiro ano de vida das crianças, e é compatível com pesquisas realizadas em outros locais (McCORMICK, 1989; BROOKS GUNN, 1990; VOHR, 1991), onde o peso ao nascer apresentou uma associação com o baixo desempenho no Teste Denver II.

\section{Considerando-se o desenvolvimento neuropsicomotor em função do estado} nutricional, peso e a estatura atuais das crianças da amostra, observou-se que não há diferença estatisticamente significativa na distribuição do percentual da variável condição nutricional e peso atuais em relação ao desempenho das crianças da amostra. O mesmo não aconteceu com a estatura atual, que se mostrou significativa quando relacionada com o desempenho das crianças da amostra.

Este resultado também confirma resultados semelhantes encontrados nas pesquisas de Cunha (2000), onde a condição nutricional não se mostrou significativa relativamente ao desempenho no Teste de desenvolvimento.

O desenvolvimento neuropsicomotor em função da renda familiar não apresentou diferença estatísticamente significativa nas crianças do presente estudo. Este resultado está de acordo com as pesquisas de Cunha (2000), mas contrastam, parcialmente, com a avaliação realizada em uma outra pesquisa de coorte em crianças de Pelotas (VICTORA et al., 1989). No estudo, o efeito da renda foi mais importante do que o do peso ao nascer como determinante de atraso. Uma possível explicação para esta variação é o diferente instrumento utilizado, o Teste de Griffits (GRIFFITS, 1970), que tem finalidade diagnóstica e não de triagem, como o teste de Denver. Entretanto, talvez a principal razão para a diferença entre estes estudos é que no da coorte de 1982 as crianças tinham idade bem superior - média de 53 meses, e se poderia esperar que, com o tempo, as variáveis relacionadas com estrutura familiar e fatores sócio-econômicos adquirissem maior importância, superando o efeito do peso ao nascer (MEISELS \& WASIK, 1990).

Foi também relacionado o desenvolvimento neuropsicomotor com o tempo de aleitamento materno exclusivo. Apesar de ser o aleitamento materno uma variável das mais 
importantes relativamente para o desenvolvimento infantil, esta não se mostrou estatisticamente significativa quando relacionada ao desempenho das crianças da amostra.

Um dado importante também contemplado no presente estudo foi a associação entre o desenvolvimento neuropsicomotor com a assistência no pré-natal e a ocorrência de patologias durante a gestação. Quanto à assistência no pré-natal $\left(\chi^{2}=10,75 ; \mathrm{gl}=3 ; \mathbf{p}=\mathbf{0 , 0 1 3}\right)$, o número de consultas pré-natais se mostrou preditor de um melhor desenvolvimento das crianças, assim como as ocorrências de patologias durante a gestação. Dessa forma, crianças cujas mães tiveram um maior número de consultas pré-natais demonstraram uma melhor performance no Teste Denver II. De maneira semelhante, crianças que não tiveram patologias durante a gestação, também apresentaram melhor desempenho no referido teste.

A escolaridade das mães com relação ao desenvolvimento neuropsicomotor se mostrou preditora de um melhor desempenho das crianças, medido através do Teste Denver II $\left(\chi^{2}=14,29 ; \mathrm{gl}=6 ; \mathbf{p}=\mathbf{0 , 0 1 6}\right)$. Estudos de Souza (2003) com a intenção de avaliar o desenvolvimento neuropsicomotor através do teste de Denver II em Cuiabá-MT, verificaram resultados semelhantes, uma vez que o desempenho das crianças se mostrou pior em famílias com escolaridade mais baixa.

A identificação precoce de problemas do desenvolvimento é uma tarefa difícil para o profissional que trabalha com cuidados primários. Devido à grande maleabilidade do desenvolvimento neuropsicomotor da criança, é necessário que a avaliação seja repetida, principalmente durante os primeiros anos de vida, quando o desenvolvimento é mais dinâmico e o impacto dos atrasos é mais importante (DWORKIN, 1989). Embora reconhecendo que as intervenções precoces em países em desenvolvimento possam ser mais difíceis de implementar (VICTORA et al, 1982), existem inúmeras alternativas para se promover programas de baixo custo e de abordagem comunitária envolvendo crianças com riscos de atraso, tais como visita domiciliar (ocasião em que a mãe é orientada a estimular seu filho), treinamento de cuidadores de crianças em creches e escolas infantis, de professores de escolas de primeiro grau, bem como o envolvimento com a mídia.

Estudos recentes demonstraram que fatores de risco múltiplos aumenta a probabiblidade do desenvolvimento da criança ser comprometido (MEISELS \& WASIK, 1990; SAMEROFF, et al., 1987). Desta forma, as crianças de países subdesenvolvidos concentram a grande mioria das possíveis causas que levam a um atraso do desenvolvimento neuropsicomotor (HALPERN et al., 1996). Chamamos a atenção para o fato de que a 
inexistência de sistemas de vigilância, dificulta a identificação e o acompanhamento das crianças vulneráveis, bem como a não utilização de instrumentos adequados para triagem de crianças com risco de atraso no desenvolvimento neuropsicomotor (TORBURN, 1990).

Outras variáveis, como a educação materna, o tamanho da família e a ocupação do pai, que neste estudo não foram abordadas, são também importantes prediteras dn desenvolvimento futuro da criança (BROMAN, 1984).

Diante dos resultados obtidos fica evidente a necessidade de se promover c apuraı iniciativas ligadas a programas comunitários de proteção infantil, principalmente em Municípios brasileiros onde ainda é predominante a escassez de recursos e a baixa escolarização.

\section{Referências:}

ALENCAR, EMLS. - Psicologia: introdução aos princípios básicos do comportamento, 5. ed.

ANDRADE, F. M. O Programa de Saúde da Família no Ceará. 1 ed. Fortaleza: 1998.

ARCHER, P; BRESNICK, B; DODDS, J; FRANKEMBURG, WK, SHAPIRO, H. - The

Denver II: a major revision and restandardization of the Denver Developmental Screening test. Pediatrics. Vol. 89. 1992.

BATSHAW, ML. Retardo mental. In: Clínicas Pediátricas da América do Norte. v.3. Rio de Janeiro: Interlivros, 1993. p. 549-564.

BRASIL, Ministério da Saúde, FIOCRUZ. Assistência perinatal e neonatal no Brasil, Tema/rodis 17, Fev. 1999.

BRASIL. Atribuições de cada membro da ESF e das ESB. Guia Prático do programa de Saúde da Família, Brasília: 76, 2001.

BROMAN, S., 1984. The collaborative perinatal project: an overview. In: Handbook of Longitudinal Research. Vol 1. Birth and Childhood Cohorts (S. Mednick, M. Harway \& K. Finelle, eds.), pp. 185-215, New York: Praeger.

BROOKS GUNN, J., 1990. Enhancing the development of young children. Current Opinion in Pediatrics, 2:873-877. 
CUIDADOS PRIMÁRIOS DE SAÚDE. Relatório conjunto do Diretor Geral da Organização Mundial de Saúde e do Diretor Executivo do Fundo das nações Unidas para a Infância. Alma Ata, URSS, 6-12 setembro de 1978.

CUNHA, H.L. Desenvolvimento de Crianças Atendidas no Hospital de Pediatria da Universidade do Rio Grande do Norte, no Primeiro Ano de Vida: Aplicação do teste de Denver II no ambulatório, dissertação de mestrado pela Universidade Federal de São Paulo- Escola Paulista de Medicina. 2000.

DARGASSIES, SS. - Long-term neurological follow-up study of 286 truly primitivy infants. In: Neurological Sequalac. Dev. Med. Child. Neurol. (1980): 462-78.

DWORKIN P.H. British and American recommen-dations for developmental monitoring: the role of surveillance. Pediatrics 1989; 84: 1000-10.

FRANKENBURG WK, DODDS J, ARCHER P, SHAPIRO H, BRESNICK B: The Denver II: a major revision and restandardization of the Denver Developmental Screening Test. Pediatrics 1992; 89: 91-7.

GLASCOE, F.P., BYRME, K.E., ASHFORD, L.G., JOHNSON, K.L., STRICKLAND, B. - Acuracy of the Denver II in developmental screening [see vommrnyd]. Pediatrics. 89 (6TT 2): 1221-5, 1997.

GRANTHAM McGREGOR, S. M.; POWEL, C.; STEWART, M. \& SCHOFIELD, W. N., 1982. Longitudinal study of growth and development of young Jamaican children recovering from severe protein-energy malnutrition. Developmental Medicine Child Neurology, 24:321-331.

GRANTHAM McGREGOR, S. M.; SCHOFIELD, W. N. \& POWEL, C., 1987. Development of severely malnourished children who received psychosocial stimulation: six-year followup. Pediatrics, 79: 247-254.

GRIFFITS, R., 1970. The Abilities of Young Children: A Comprehensive System of Mental Measurement for the First Eight Years of Life. London: Child Development Research.

HALFON N, LONG PV, ZERDEN M: Pediatrics, health development, and the third revolution in health care delivery. In Hager M (Ed.), Pediatric Education in the 21st 
Century: Proceedings of a Conference Chaired by Barry Zuckerman, MD. New York, NY: Josiah Macy, Jr. Foundation, 2004: pp. 131-154.

HALPERN, R; SHAEFER, ES; PEREIRA, AS; ARNT, EM; BEZERRA, JPV; PINTO, LS. Fatores de risco para baixo peso ao nascer em uma comunidade rural no sul do Brasil. Jornal de Pediatria, 72 (6): 369-378, 1996.

HALPERN, R.; BARROS, F.C.; HORTA, B.L.; VICTORA, C.G. Desenvolvimento neuropsicomotor aos 12 meses de idade em uma coorte de base populacional no Sul do Brasil: diferenciais conforme peso ao nascer e renda familiar. Cad. Saúde Pública v.12 supl.1 Rio de Janeiro 1996.

KING, E.H., LOGSDON, D.A., SCHOROEDER, R,S. Risk factores for developmental delay among infants and toddlers. Child Healt Care, 2004. 21: 39-52.

LEÃO, E. e outros. - Pediatria Ambulatorial. 3. ed. Belo Horizonte: Cooperativa Editora e Cultura Médica, 1998.908 p.

LEVY, SE; HYMAN, SL. - Avaliação pediátrica da criança com retardo do desenvolvimento. In: Clínicas Pediátricas da América do Norte, v. 3. Rio de Janeiro: Interlivros, 1993. p. 501-513.

McCORMICK, M. C., 1989. Long-term follow-up of infants discharged from neonatal intensive care units. Journal of American Medical Association, 261:1767-1772.

MADEIRA, LM. - Alta Hospitalar da Criança: Revista Brasileira de Crescimento e Desenvolvimento. Ano IV, n.2, 1994, Faculdade de Saúde Pública da USP.

MARCONDES, E. - Desenvolvimento da Criança: Desenvolvimento biológico, crescimento. Rio de Janeiro: Sociedade Brasileira de Pediatria, 1994. 78 p.

MEISELS, J. S. \& WASIK, B. A., 1990. Who should be served? Identifying children in need of early intervention In: Handbook of Early Intervention (S. J. Meisels \& J. Shonkoff, eds.), pp. 605-632, Cambridge: Cambridge University Press.

MINISTÉRIO DA SAÚDE. Pesquisa Nacional de Demografia e Saúde 1996 - PNDS/96.

Brasília: Sociedade Civil do Bem-estar Familiar no Brasil - BEMFAM; 1997.

NELSON, WE e cols. - Tratado de Pediatria. 4 ed. Salvat Editores S/A: Barcelona - Madrid, 1960. 
NICOLL A, HUTCHINSON E, SOLDAN K, HEPTONSTALL J, PARRY J, NEWHAM J, et al. Survey of human immunodeficiency virus infection among pregnant women in England. Results of the first four years (1990-93). Communicable Disease Reports (in press).

SAIGAL, S.; SZATMANI, P.; ROSEMBAUM, P.; CAMPBELL, D.; KING S., 1991. Cognitive abilities and school performance of extremely low birth weight children and matched term control children at age 8 years: a regional study. Journal of Pediatrics, 118:751-760.

SAMEROFF, A.J.; SEIFER, R.; BAROCAS, P.B.; ZACK, M.; GREENSPAN, S. IQ scores for 4-year-old children: social environmental risk factors. Pediatrics, 79: 343-50, 1987.

SIMEONSSON, R. J.; COOPER, D. H. \& SCHEINER, A. P., 1982. A review and analysis of the effectiveness of early intervention programs. Pediatrics, 69: 635-641.

SOUZA, S. C. Avaliação do desenvolvimento neuropsicomotor do pré-escolar de creches públicas de Cuiabá-MT, 2003. Tese de doutorado em Pediatria. São Paulo: Universidade de São Paulo - USP, 2003.

TORBURN, J. M., 1990. Childhood disability in developing countries: basic issues. In: Practical Approaches to Childhood Disability in Developing Countries: Insights from Experience and Research (J. M. Thorburn \& J. Marfo, eds.), pp. 3-28, St John's: Project

VICTORA, C.G.,BARROS, F.C., MARTINES, J.C., BÉRIA, J.U., VANGHAN, J.P. Estudo longitudinal das crianças nascidas em 1982, em Pelotas, RS - Brasil: Metodologia e resultados preliminares. Rev. Saúde Pública v.19, n.1. São Paulo, fev.1985.

VICTORA, C. G.; BARROS, F. C. \& VAUGHAN, J. P., 1989. Epidemiologia da Desigualdade. $2^{\mathrm{a}} \mathrm{ed}$., São Paulo: Hucitec.

VOHR, R. B., 1991. Preterm cognitive development: biologic and environmental influences. Infant and Young Children, 3:20-29.

VOHR, R. B., 1991. Preterm cognitive development: biologic and environmental influences. Infant and Young Children, 3:20-29. 